\title{
EVALUATING SPECIES INTERACTIONS AS A DRIVER OF PHYTOPHAGOUS INSECT DIVERGENCE
}

\author{
Bruno A. S. de Medeiros ${ }^{1,2}$ and Brian D. Farrell ${ }^{2}$ \\ ${ }^{1}$ Smithsonian Tropical Research Institute \\ Balboa Ancón, Panama City, Panama \\ demedeirosb@si.edu
}

${ }^{2}$ Museum of Comparative Zoology, Department of Organismic \& Evolutionary Biology, Harvard University

Cambridge, MA, United States

\section{ABSTRACT}

Plants and their specialized flower visitors provide valuable insights into the evolutionary consequences of species interactions. In particular, antagonistic interactions between insects and plants have often been invoked as a major driver of diversification. Here we use a tropical community of palms and their specialized insect flower visitors to test whether antagonisms lead to higher population divergence. Interactions between palms and the insects visiting their flowers range from brood pollination to florivory and commensalism, with the latter being species that feed on decaying and presumably undefended plant tissues. We test the role of insect-host interactions in the early stages of diversification of nine species of beetles sharing host plants and geographical ranges by delimiting cryptic species and then using models of genetic isolation by environment. The degree to which insect populations are structured by the genetic divergence of plant populations varies. A hierarchical model reveals that this variation is largely uncorrelated with the kind of interaction, showing that antagonistic interactions are not associated with higher genetic differentiation. More general aspects of host use that affect plant-associated insects regardless of the outcomes of their interactions, such as sensory biases, are likely more general drivers of insect population divergence. 


\section{INTRODUCTION}

Insects comprise about two thirds of the 1.5 million described species of animals [1], and current estimates predict that another 4 million insect species remain unknown [2]. This spectacular diversity is thought to be in a large degree a consequence of ecological speciation resulting from interactions with plants, particularly antagonistic interactions [3-11]. Antagonism between plants and insects could lead to accelerated rates of diversification, with the diversity of defenses among plants resulting from host specialization that in turn may spur radiations in insects circumventing those defenses [7,8,11-14]. The effects of these interactions are not restricted to macroevolution: theoretical models predict that, in specialized interactions, coevolution can lead to stronger differentiation when compared to spatial isolation alone in the case of antagonism but not in mutualism $[15,16]$. Regardless of the proximal mechanisms, a pattern of strong isolation by environment [17] may be expected when insect-plant interaction is a major cause of reduced gene flow and an insect species interacts with different plant species or populations. For example, in brood pollinators (specialized pollinators that are also seed predators [18]) it has been observed that more divergent host plant populations are associated with more divergent insect populations [19-24], but not in all cases evaluated [24,25].

If antagonisms promote divergent selection leading to the formation of host races and ecological speciation [10], genetic isolation between plant populations may be a better predictor of insect isolation in antagonists than in mutualists or commensals. It is unclear whether this is the case for most plant feeding insects, especially considering that these interactions often involve multiple partners and are spatially and temporally variable and context-dependent [26]. Here we test this prediction by using a direct comparison between insects with different modes of interaction across scales of plant divergence. We take advantage of the variation in insect-plant interactions found in communities of palm-associated weevils distributed across the same geographic range and interacting with the same plants. We specifically test the hypothesis that isolation associated with host plant divergence is stronger in antagonistic species when compared to isolation by geographical distance alone.

Palms in the genus Syagrus, one of the closest relatives of the coconut $[27,28]$, produce large inflorescences that are visited by dozens of insect species [29-33]. The most abundant flower visitors of these Neotropical palms are specialized beetles in the family Curculionidae, one of the most diverse insect taxa [34]. We recently described the community of insects associated with the seasonally dry forest palm Syagrus coronata, showing that many weevil species are broadly distributed throughout the plant geographical range [32]. Some of them are brood pollinators, while others are antagonists breeding on flowers or seeds and some are commensals breeding on decaying plant tissues. Populations of Syagrus coronata have been found to have deep genetic divergences [35], and this plant shares many species of weevil with $S$. botryophora, a parapatric palm specialized on rainforests and diverged from $S$. coronata early in the history of the genus, about 20 million years ago [27,28]. Given this old divergence, weevil morphospecies shared by the two plants are likely a result of relatively recent host shifts as opposed to long-term co-diversification. We used double- 
digest RADseq (ddRAD), a low cost genome-wide sequencing method [36,37], to obtain genome-wide genetic markers for several populations of both plant species, including a population of $S$. coronata known as $S$. $\times$ costae, a hybrid with $S$. cearensis [27]. We used the same method to sequence nine morphospecies of weevil broadly distributed across the range of these palms (Figure S1). These nine morphospecies are all attracted to flowers and locally specialized on their host plants. They mate and lay eggs on their hosts and are distributed through a similar geographical range, but differ in the kind of interaction with plants in two relevant axes: their roles as pollinators as adults and whether their larvae breed on live or decaying tissues (Table 1). We first use the genomic data to delimit weevil species and better understand the diversity of these littleknown insects. Then, we test models of isolation by environment to ask whether the kind of interaction with host plants is associated with differences in the degree of isolation by geographical distance or isolation associated with host plant genetic divergence. Finally, we fit a Bayesian hierarchical model to test whether species with antagonistic interactions exhibit stronger levels of host-associated differentiation in relation to other species.

Table 1 Weevil morphospecies included in the study with references for natural history information.

\begin{tabular}{|c|c|c|c|}
\hline Morphospecies & Pollinator & Larval breeding & Host palms \\
\hline $\begin{array}{l}\text { Anchylorhynchus } \\
\text { trapezicollis[32] }\end{array}$ & Yes & Live tissue (developing fruits) & both \\
\hline Remertus rectinasus[32] & No & Live tissue (developing fruits) & both \\
\hline Microstrates bondari[38] & No & Live tissue (male flower buds) & both \\
\hline Microstrates ypsilon[32] & No & Live tissue (male flower buds) & S. coronata \\
\hline $\begin{array}{l}\text { Andranthobius } \\
\text { bondari[32] }\end{array}$ & No & $\begin{array}{l}\text { Decaying tissue (aborted male } \\
\text { flowers) }\end{array}$ & both \\
\hline Celetes impar[32] & No & $\begin{array}{l}\text { Decaying tissue (peduncular bract } \\
\text { after anthesis) }\end{array}$ & S. coronata \\
\hline Celetes decolor[32] & No & $\begin{array}{l}\text { Decaying tissue (floral branches after } \\
\text { fruit dispersal) }\end{array}$ & both \\
\hline Dialomia polyphaga[32] & No & $\begin{array}{l}\text { Decaying tissue (damaged } \\
\text { inflorescences) }\end{array}$ & S. coronata \\
\hline $\begin{array}{l}\text { Phytotribus } \\
\text { cocoseae[39,40] }\end{array}$ & No & $\begin{array}{l}\text { Decaying tissue (peduncular bract } \\
\text { after anthesis) }\end{array}$ & $\begin{array}{l}\text { S. } \\
\text { botryophora }\end{array}$ \\
\hline
\end{tabular}

\section{RESULTS}

\section{CRYPTIC WEEVIL SPECIES}

We initially assembled genomic datasets by filtering low-coverage loci $(<12$ reads) and genotyping each individual separately. Visualization of patterns of missing data revealed that, for some of the weevil species, certain ddRAD loci are shared within groups of samples, with very few loci recovered across groups (Figure S2). This pattern could be an artifact resulting from batch effects during ddRAD library preparation, because samples in a batch are pooled before size selection and PCR amplification [36]. Alternatively, it could be a consequence of cryptic, deeply differentiated taxa contained 
within each species as traditionally recognized by morphology [41]. Since studying the early stages of divergence does not make sense in the complete absence of gene flow $[10,42]$, we first evaluated whether our dataset included cryptic species.

To test whether this is the case, we recorded the number of loci shared, average sequence divergence and batch identity for each pair of samples in each morphospecies. We found that samples processed in the same batch do share more loci, but extreme levels of missing data are only explained by deep sequence divergence, sometimes above 2.5\% (Table 2, Figure S2). We note that, in all cases, splitting samples into operational taxonomic units (OTU) at this level of sequence divergence results in groups with very high genetic differentiation from each other as measured by G'st (Figure S2). With the exception of Anchylorhynchus trapezicollis, these clusters separate populations on each host plant (Figure 1). For all kinds of interactions, there is negligible to zero gene flow between these populations on the two different host plant species. In the case of the pollinator Anchylorhynchus trapezicollis, we find three genetic clusters, with one of them in both host species and broadly sympatric with the other two (Figure 1). By comparing the morphology of the two most abundant clusters in sympatry and allopatry, we found differences in the length of ventral plumose hairs and in male secondary sexual characters (Figure S3). These diverged genetic clusters represent cryptic, previously unrecognized species. Hereafter, we will use OTUs as our unit of analysis, noting that these will be properly described as new species in the future. In general, we also recommend caution in studies of little known organisms in which cryptic species might be common [43], noting that we were only able to distinguish OTUs because samples were individually barcoded and not pooled by location.

Table 2 Effect of nucleotide distance and shared library batch on number of shared RAD loci (thousands). All samples of $R$. rectinasus were prepared in the same batch. ${ }^{*} p$-value $<0.01$

\begin{tabular}{lllll} 
Morphospecies & Intercept & Distance & Batch & $\mathbf{R}^{2}$ \\
\hline $\begin{array}{l}\text { Anchylorhynchus } \\
\text { trapezicollis }\end{array}$ & $7.4^{*}$ & $-1.5^{*}$ & 0.3 & 0.46 \\
Andranthobius bondari & $3.2^{*}$ & $-0.8^{*}$ & $0.7^{*}$ & 0.15 \\
Celetes decolor & $3.7^{*}$ & $-0.6^{*}$ & 1.0 & 0.15 \\
Celetes impar & 7.2 & -0.4 & 0.2 & 0.002 \\
Dialomia polyphaga & 4.1 & -2.9 & 0.6 & 0.09 \\
Microstrates bondari & 1.6 & 0.4 & $1.4^{*}$ & 0.08 \\
Microstrates ypsilon & 3.8 & -0.7 & 0.1 & 0.01 \\
Phytotribus cocoseae & $18.5^{*}$ & $-24.2^{*}$ & $1.4^{*}$ & 0.21 \\
Remertus rectinasus & 3.4 & $-0.6^{*}$ & - & 0.04 \\
\hline
\end{tabular}

A principal component analysis of the genetic variation of each OTU reveals little spatial congruence among weevil OTUs and variable congruence with the genetic variation of their host plants (Figure 1). We found evidence for genetic clusters in 12 of the 13 
weevil OTUs (Figure 1, Figure S4) and investigated whether there is gene flow between these clusters by using a model of isolation-with-migration based on the site frequency spectrum (Figure S5). We found that, in all cases, models including migration had higher support than those that did not (Table 3, Table S1). Populations of Anchylorhynchus trapezicollis OTU 1 and Remertus rectinasus on different host plants have much deeper divergence and smaller migration rates than those interacting with Syagrus coronata alone (Table 3, Table S1), indicating that there are well-delimited host races even in these cases that divergence is shallow enough to enable assembly of ddRAD datasets.
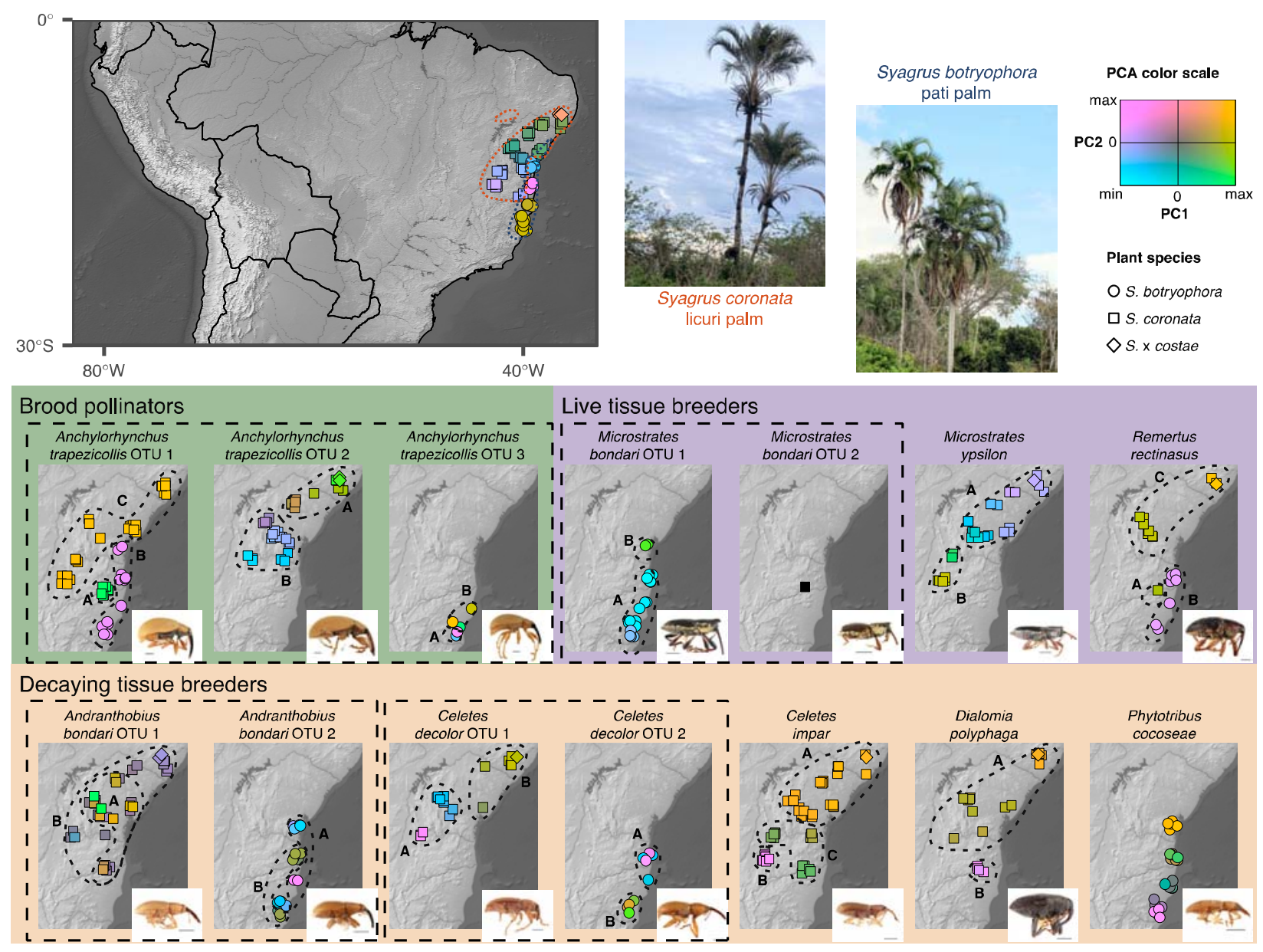

Figure 1 Principal component analyses (PCA), done separately for each plant species and insect OTU. In each case, the position of a sample in the first two PC axes is coded following the color legend provided: samples with similar colors have similar PCA scores. M. bondari OTU 2 is black since no PCA is possible with a single sample. Figure S4 shows the same PCA results but plotted in traditional coordinates instead of colors in a map. A small jitter was added to enable visualization of overlapping points. Dashed boxes enclose morphospecies. Large map includes known palm distributions[27,44] enclosed in dashed lines. PCAs are independent for S. botryophora 
and S. coronata. Small maps show PCA results for each weevil OTU, with clusters enclosed in black dashed lines and labelled with uppercase letters being used in coalescent models (Table 3, Table S1). Scales $1 \mathrm{~mm}$ in insect images.

Table 3 Summary of isolation-with-migration model fit, showing pairs of populations with direct gene flow inferred in the best and second best model, as well as the $\triangle \mathrm{AIC}$ between them. Population labels follow Figure 1, full table with inferred parameters in Table S1.

\begin{tabular}{lllll}
\hline OTU & $\begin{array}{l}\text { Populations } \\
\text { included }\end{array}$ & $\begin{array}{l}\text { Populations } \\
\text { connected } \\
\text { in best } \\
\text { model }\end{array}$ & $\begin{array}{l}\text { Populations } \\
\text { connected } \\
\text { in second } \\
\text { best model }\end{array}$ & \\
\hline Anc. trapezicollis OTU 1 & 3 & $\mathrm{AB}, \mathrm{AC}, \mathrm{BC}$ & $\mathrm{AC}, \mathrm{BC}$ & 1848 \\
Anc. trapezicollis OTU 2 & 2 & $\mathrm{AB}$ & none & 5645 \\
Anc. trapezicollis OTU 3 & 2 & $\mathrm{AB}$ & none & 228 \\
And. bondari OTU 1 & 2 & $\mathrm{AB}$ & none & 6273 \\
And. bondari OTU 2 & 2 & $\mathrm{AB}$ & none & 2886 \\
C. decolor OTU 1 & 2 & $\mathrm{AB}$ & none & 3656 \\
C. decolor OTU 2 & 2 & $\mathrm{AB}$ & none & 182 \\
C. impar & 3 & $\mathrm{AB}, \mathrm{AC}, \mathrm{BC}$ & $\mathrm{AB}, \mathrm{BC}$ & 1080 \\
D. polyphaga & 2 & $\mathrm{AB}$ & none & 902 \\
M. bondari OTU 1 & 2 & $\mathrm{AB}$ & none & 1096 \\
M. ypsilon & 2 & $\mathrm{AB}$ & none & 2620 \\
R. rectinasus & 3 & $\mathrm{AB}, \mathrm{AC}$ & $\mathrm{AB}, \mathrm{AC}, \mathrm{BC}$ & 15 \\
\hline
\end{tabular}

\section{INTERACTIONS DO NOT PREDICT PATTERNS OF ISOLATION}

Following evidence for ongoing gene flow between populations in each OTU, we assessed the role of geography and plant host as genetic barriers for each species of weevil. We also include climate in this analysis to account for the possibility of other differences in environment acting as genetic barriers. We used matrices of geographical distance, host plant genetic distance and climatic distance between weevil populations as explanatory variables for the genetic covariance between weevils in a Bayesian model of isolation by distance and environment $[45,46]$. With model choice by cross validation, we found that climate was not a significant barrier to gene flow for any weevil species, and the significance of geography or host plant varied (Table S2). For this reason, we ran these models again using the full dataset, but including only geography and host plant as predictors. The importance of geography or host plant as the main driver of divergence varied between weevil OTUs, and this variation seems uncorrelated to the mode of interaction (Figure 2, Table S3). 

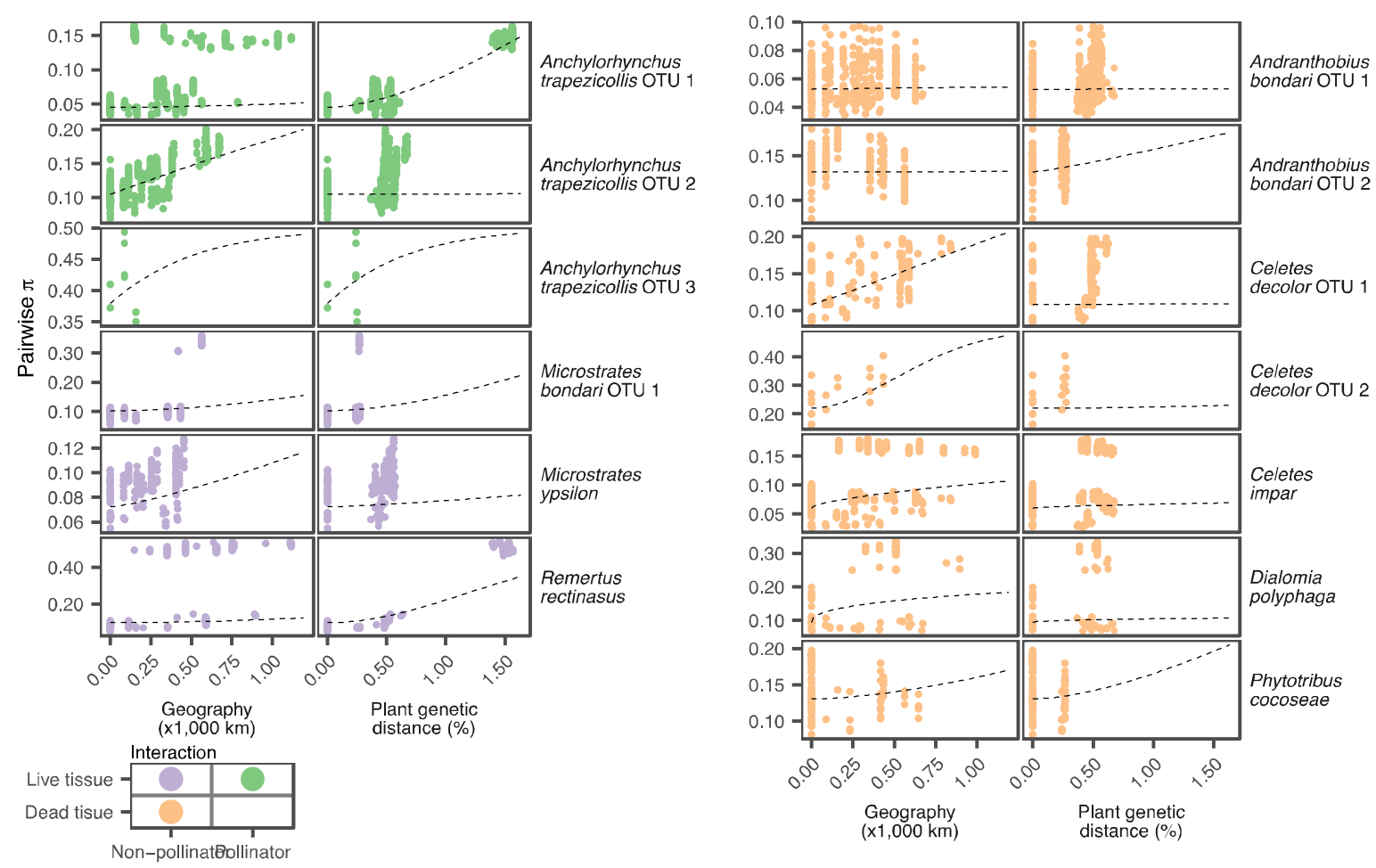

Figure 2 Effects of the geographical and plant distance on weevil pairwise distance in SNP sites (pairwise $\pi$ ). Dashed lines show the marginal effects of each distance implied by average parameter estimates.

To test whether species interactions are associated with differences in patterns of genetic divergence, we calculated the statistics $\alpha_{\text {diff }}$, which describes the relative importance of host plants as sources of population divergence when compared to geography for a given OTU (see Methods). We then implemented a hierarchical Bayesian model to evaluate the independent effect of being a pollinator or breeding on live tissue (i. e. being an antagonist) on the value of $\alpha_{\text {diff }}$ (eq. 1, eq. 2). We scored interactions along these two independent axes because the positive aspect of a brood pollination interaction may also affect rates of population divergence. Mutualisms have sometimes been claimed to lead to highly specialized interactions and thereby promote diversification in both insects and plants [47-49], but theoretical models do not predict that mutualisms lead to divergence in specialized interactions [16]. We note that Anchylorhynchus weevils are not exclusive pollinators of species of Syagrus palms $[31,32,50]$ and the net effect of these interactions are currently unknown. Our model estimates the effect of pollination or antagonism by the parameters $\gamma_{p o l}$ and $\gamma_{\text {ant }}$, respectively (eq. 2). A significantly positive value for these parameters means that pollinators $\left(\gamma_{p o l}\right)$ or antagonists $\left(\gamma_{\text {ant }}\right)$ experience higher levels of divergence related to host plant divergence when compared to geography alone. We used posterior predictive simulations to find that the model adequately fits to the data (Figure S7), and also found that the number of OTUs used in this study provides enough power for inferences (see 
methods) (Figure S8). There is substantial variation in estimated $\alpha_{\text {diff }}$ between OTUs (Figure 3A), but no evidence that $\gamma_{p o l}$ is significant on either direction (Figure 3B). While there is a positive trend for $\gamma_{a n t}$, its $95 \%$ credibility interval includes negative values (Figure 3B).

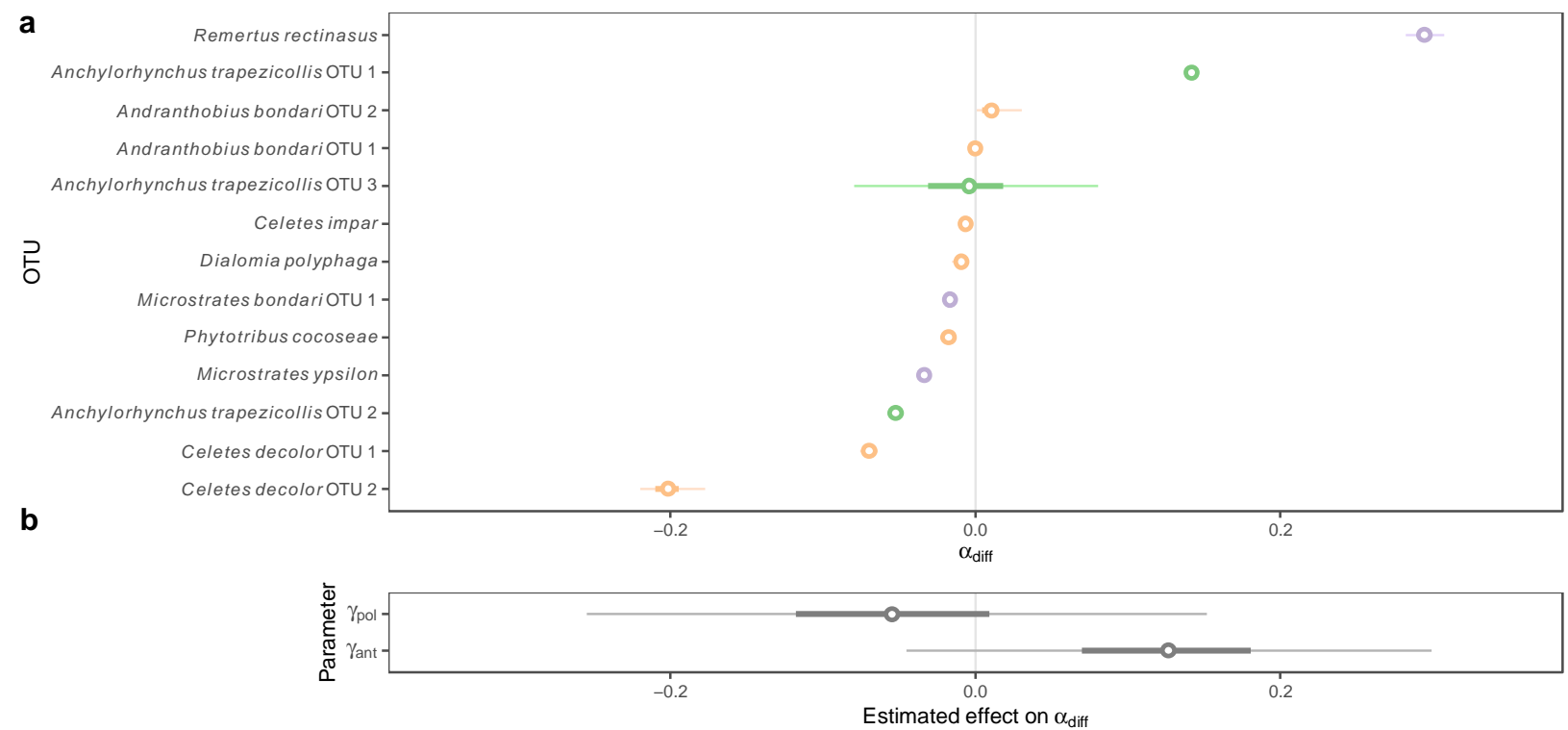

Figure 3 Effects of insect-plant interaction on variation of $\alpha_{\text {diff }}$ across OTUs. Points: average estimates, thick lines: $50 \%$ credibility intervals, thin lines: $95 \%$ credibility intervals. a) Posterior distribution of $\alpha_{\text {diff }}$ across OTUs, ordered by average $\alpha_{\text {diff }}$.b) Posterior distribution of $\gamma$ parameters.

\section{DISCUSSION}

The degree of isolation by distance and by environment in these weevils co-distributed throughout the same range and interacting with the same plants varies widely, and this variation is largely unrelated to the kind of interaction with their hosts. All insect morphospecies previously thought to interact with both of two host plant species are actually comprised of cryptic species or highly divergent populations, each specialized on a single host plant species. This is evidence that host plants constitute an important barrier for all beetle species sampled here. At a finer scale, plant host population divergence is a barrier to weevil gene flow for a subset of weevil OTUs, encompassing all kinds of interactions. OTUs breeding on live plant tissue seem to experience slightly higher divergence associated with host plants, but not significantly higher than other OTUs. Closely related OTUs do not necessarily show similar responses to geography and host plant divergence, suggesting that phylogenetically conserved traits (such as lifespan or flight ability) are not major drivers of the differences observed.

The lack of effect of pollination does not imply that mutualisms in general do not affect insect divergence rates. Anchylorhynchus weevils are not exclusive pollinators of Syagrus and therefore the outcomes of these interactions are very likely to be context- 
dependent and geographically variable, as other cases of non-specialized brood pollination [51]. Moreover, the difference in morphology of ventral hairs between OTUs might be related to pollen-carrying capacity. The lack of effect of antagonism, however, is unexpected. Palm flowers have chemical and physical defenses against herbivory [52], but weevil OTUs breeding on decaying tissues and therefore not interacting with these defenses exhibit similar patterns of isolation to those that do attack live defended tissues. While the ability to digest and detoxify plant tissues is thought to be a key adaptation enabling macroevolutionary diversification of phytophagous beetles [53], and weevils specifically [54], it unlikely that coevolution and adaptation to plant defenses is a universal source of divergent selection and a necessary condition to explain the high rates of insect speciation. A recent review found that most studies on candidate genes for specialization to hosts in phytophagous insects focus on resistance or detoxification of plant secondary metabolites [55], but the actual source of selection might be in other aspects of host use. Divergence following host shifts is pervasive in phytophagous insects and their parasitoids [56], despite the large variation in interaction outcomes. Even though coevolution is an important driver of diversification under some conditions $[6,8,16,48,57,58]$, evolution without reciprocal adaptation might be sufficient to explain many or most cases of insect specialization.

Diverse and complex phytophagous insect communities such as the one we study here are likely the norm rather than the exception in insect-plant interactions. Here we found that all weevil species, including those breeding on decaying plant tissues, show similar patterns of host-associated divergence. Strict antagonistic coevolution and divergence of host plant defenses are unlikely to drive this pattern. Despite the variation in larval breeding sites, all of the weevil species evaluated here mate on flowers [32], and it is possible that the usage of flowers as mating signals is a more general driver of divergence for these beetles and other phytophagous insects. Verbal models of how the evolution of sensory biases could be a major driver of phytophagous insects diversification have been proposed for a long time [59,60], but have received little attention in comparison to the wealth of research focused on plant defenses as drivers of diversity spurred by the classic study of Ehrlich and Raven [13]. The evolution of odorant receptors associated with mating signals in insect flower visitors has been recently linked to species divergence in at least one case [61]. Considering that about one third of insect species visit flowers [62], the generality of flowers and other host plant cues working as mating signals that result in insect species divergence should receive more attention.

We studied patterns of isolation by distance and by environment in nine morphospecies of weevils associated with flowers of two palm species, which turned out to be 14 weevil OTUs after cryptic species were identified. Host plant species identity was a very strong barrier to gene flow in all cases, with a different OTU or a highly divergent population on each host. Both geography and host plants, but not climate, are important barriers determining genetic differentiation, with variation between insect species being largely unrelated to the kind of interaction with their host plants. Insectplant antagonistic coevolution does not seem to be required for insect specialization and the generation of barriers to gene flow, and other aspects of insect-host interactions, 
such as sensory biases, should be investigated in studies of phytophagous insect diversification.

\section{METHODS}

\section{SAMPLING}

We sampled insects and plants from 13 populations of Syagrus coronata (including S. $\times$ costae, hybrids with S. cearensis [27]) and five populations of Syagrus botryophora throughout the distribution of both plant species (Figure 1). Whole inflorescences were bagged and excised, with insects aspirated and stored in 95\% ethanol. Leaf tissues were collected from the sampled plant and other individuals in the vicinity. For this study, we chose nine specialized weevil species that we previously identified to engage into different kinds of interaction with their host plants and which have widespread geographical distributions[32] and sequenced 1-10 individuals per morphospecies per locality (Figure S1).

\section{DNA EXTRACTION AND LIBRARY PREPARATION}

We extracted DNA from insects and prepared double-digest RAD-seq libraries [36] from $150 \mathrm{ng}$ of input DNA as described in de Medeiros \& Farrell [37], including wholegenome amplification for low-yield DNA extracts. Some of the individuals were extracted destructively, but for others we digested full bodies split at the base of the pronotum and preserved the remaining cuticle. For plants, DNA was extracted from leaf tissues using the E.Z.N.A. HP Plant DNA Mini Kit (Omega Biotek) following the manufacturer protocol and libraries were prepared with the same enzymes and protocol as for insects, but from 300-1000 ng of genomic DNA without whole-genome amplification. Barcoded libraries were sequenced on Illumina systems, in several runs pooled with unrelated libraries. The minimum sequence length was single-end $100 \mathrm{bp}$, and all sequences were trimmed to this length prior to assembly.

\section{INITIAL DATASET ASSEMBLY}

Sequences were demultiplexed by inline barcodes and assembled using ipyrad v.0.7.24 $[63,64]$. For insects, sequences were entirely assembled de novo, but removing reads of potential endosymbionts by using the ipyrad option denovo-reference with reference sequences including genomes of known weevil symbionts [65] as well as Rickettsia and Wolbachia genomes downloaded from the NCBI. We assembled datasets separately for each insect morphospecies. For plants, sequences were assembled either by mapping to the draft genome assembly of the coconut [66] or denovo for unmapped reads, using the ipyrad option denovo+reference. Reads were clustered within and between samples at $85 \%$ identity, and only loci with coverage greater or equal than 12 in a sample were retained for statistical base calling using ipyrad. Initially, we retained all samples and all loci present in at least four samples and we used Matrix Condenser $[37,67]$ to visualize patterns of missing data. We then removed samples with excessive missing data from the datasets, since with whole-genome amplification these are more likely to include contaminants and amplification artifacts [37]. Instead of choosing an arbitrary threshold 
for filtering, we flagged for removal outliers as observed in the histogram view of Matrix Condenser.

\section{ASSESSING MISSING DATA}

For each insect morphospecies, we calculated the following pairwise metrics: (1) number of loci sequenced in common for each pair of samples, (2) the average pairwise nucleotide distance using the function dist.hamming in $\mathrm{R}$ package phangorn v.2.4.0 [68], and (3) whether the two samples were prepared in the same batch. We tested whether sequence distance and batch effects are negatively associated with the number of common loci by fitting a regression on distance matrix $[69,70]$ implemented in the $\mathrm{R}$ package ecodist v.2.0.1 [71].

\section{ASSEMBLY OF FINAL DATASETS}

After confirming that sequence distance is negatively associated with number of shared loci, we split the datasets for each morphospecies into clusters separated by at least 2.5\% nucleotide differences using the R package dendextend v.1.8.0 [72]. To further confirm if clusters thus obtained consist of highly isolated populations, we used the $R$ packages mmod v.1.3.3 [73] and adegenet v.2.1.1 [74,75] to calculate G'st [76] between these clusters using all loci present in at least one individual per cluster. In the case of Anchylorhynchus trapezicollis, clusters were sympatric across a broad range, so we compared the morphology of individuals with preserved cuticle to confirm their divergence with an independent source of data. Sequencing statistics are available in Table S4.

\section{POPULATION STRUCTURE}

We used bwa-mem v.0.7.15 [77] to map reads on the consensus sequence for each RAD loci in the final dataset. Alignment files in bam format were used as input to ANGSD v.0.920[78] and PCAngsd v.0.973 [79] to filter sites not in Hardy-Weinberg equilibrium (HWE) while accounting for population structure [80]. We removed the whole RAD locus if any site was found not to be in HWE. We then used the same software to estimate genetic covariance matrices for each insect and plant species, as well as posterior genotype probabilities. Principal component analyses (PCA) based on these covariance matrices were clustered by the k-means method with scripts modified from the $\mathrm{R}$ package adegenet. For each insect species, the optimal number of clusters was chosen by minimizing the Bayesian Information Criteria (BIC) [81].

\section{ISOLATION WITH MIGRATION MODELS}

We used ANGSD and dadi v.1.7.0 [82] to generate the multidimensional site frequency spectra for each morphospecies with more than one k-mean cluster. We used these as input for models of isolation-with-migration [83] (Figure S5) in fastsimcoal v.2.6.0.3 $[84,85]$. All simulations were done with a mutation rate of $3 e-9$, in line with other insects [86], but inferred parameters were finally scaled by the mutation rate (Figure S5). For each model, we ran 100 independent searches of the maximum likelihood parameters and selected the best model by the Akaike Information Criterion (AIC). 
We used BEDASSLE v.2.0-a1 $[45,46]$ to infer the effects of geographical distance and host plant genetic distance on the genetic covariance of weevil populations. We additionally tested the effect of climatic distance as a confounder. We generated valid [87] (i. e. Euclidean) distances for explanatory variables as follows. We projected collection localities to UTM Zone 24S using the R package sf v.0.8-0 [88] and calculated the Euclidean distance between them to obtain geographical distances. For climatic distance, we downloaded records of $S$. coronata and $S$. botryophora from GBIF [44] using the $R$ package rgbif v.1.3.0 [89], cleaned them with the R package CoordinateCleaner v.2.0-11 [90] and then used the R package raster v.3.0-7 [91] to extract bioclimatic variables [92] for these localities. We used PCA to find that the first PC explained $90.9 \%$ of the variance in the dataset and Annual precipitation (bio12) had a very high loading on this component (Figure S6). Therefore, we used the difference in Annual Precipitation as climatic distance. For plant host genetic distances, we used NGSdist v.1.0.8 [93] to estimate genetic distances between all samples of Syagrus based on posterior genotype probabilities and including invariant sites. We then calculated pairwise genetic distances between populations as the average distance between all of their samples, and checked that the resulting distances were Euclidean by using the R package ade4 v.1.7-15 [94]. For each weevil OTU with 3 or more populations sampled, we called genotypes with posterior probability $\geq 0.8$ and filtered the dataset to one site per RAD locus to avoid linked sites, including only sites genotyped in at least one sample per population. For cross-validation, we split datasets in 10 partitions with 50 replicates and chose the simplest model among those with highest explanatory power. After finding that climate was not an important variable for any species, we ran BEDASSLE2 models on the full dataset with only host plant and geography as distance matrices, with 4 chains of 2,000 generations each and used the R package shinystan v.2.5.0 [95] to evaluate convergence.

The BEDASSLE model estimates parameters associated with the strength of the relationship between a given distance matrix and the genetic isolation of species [45]. Here we denote $\alpha_{g}$ as parameter associated with geographical distance and $\alpha_{p}$ the parameter associated with host plant genetic distance. We used all samples from the posterior distribution to calculate $\alpha_{\text {diff }}=\alpha_{p}-\alpha_{g}$ for each OTU. The variation of $\alpha_{\text {diff }}$ between OTUs indicates the degree to which plant or geographical distances are associated with barriers to gene flow for each OTU, with more positive values associated with greater importance of host plants. We estimated the determinants of variation in $\alpha_{\text {diff }}$ across species by implementing a Bayesian hierarchical model similar to those typically used in meta-analyses. For each OTU j:

$$
\begin{gathered}
\alpha_{\text {diff }_{j}} \sim \operatorname{Normal}\left(\theta_{j}, \sigma_{j}\right) \\
\theta_{j} \sim \operatorname{Normal}\left(\mu+\gamma_{a n t} \times I_{a n t_{j}}+\gamma_{p_{\text {ol }}} \times I_{p_{o l_{j}}}, \tau\right)
\end{gathered}
$$

In this model, $\sigma_{j}$ is the standard deviation in the posterior estimates for $\alpha_{\text {diff }}$, calculated from BEDASSLE posterior draws and assumed as known. $\mu$ is the mean $\alpha_{\text {diff }}$ for all OTUs, estimated by the model, and $\tau$ is the estimated variation in $\alpha_{\text {diff }}$ that is unrelated to species interactions. $I_{a n t}$ and $I_{p o l}$ are indicator variables for whether 
each species is an antagonist (i. e. breeds on live tissue) or pollinator, respectively (Table 1). In our dataset, both indicators have a value of 1 for brood pollinators and 0 for non-pollinators breeding on dead tissue, while for non-pollinators breeding in live tissue $I_{\text {ant }}=1$ and $I_{p o l}=0$. The parameters $\gamma_{\text {ant }}$ and $\gamma_{\text {pol }}$, therefore, are associated with the strength of the linear relationship between $I_{a n t}$ and $I_{p o l}$ and $\alpha_{\text {diff }}$, and constitute the model output of interest here. Values significantly different from 0 indicate that antagonism or pollination has a significant effect in determining the strength of weevil population divergence imposed by host divergence, when compared to space alone. We used standard Normal priors for $\gamma_{a n t}, \gamma_{p o l}$ and $\tau$ and $\mu$. We implemented this model in rstan v.19.2[96] using the Stan language. Models were run and convergence checked as for BEDASSLE models. We also tested model fit by using posterior predictive simulations. Finally, we assessed whether the number of species included in this study is sufficient to achieve power to estimate $\gamma_{a n t}$ and $\gamma_{p o l}$ by running a model with an extreme case based on real data. We used the real distributions of $\alpha_{\text {diff }}$ but relabeled the three species with highest values as non-pollinator antagonists, the next three as both pollinators and antagonists and the remaining seven as neither pollinators or antagonists. This preserved the number of species for each category in the real data but maximized the differences in $\alpha_{\text {diff }}$ between modes of interaction.

\section{ACKNOWLEDGEMENTS}

We thank Harri Lorenzi and Larry Noblick for palm location and identifications. Curators Renato de Melo Silva (SPF) and Sonia Casari (MZSP) allowed collection access and deposit of specimens. Sergio A. Vanin assisted with weevil specimens and identification. Tauana Cunha, Elton Antunes, Filipe Gudin, Gabriel Pimenta, Zhengyang Wang, Luiz Fonseca, José I. L. Moura, Francisco José de Paula and the Queiroz family assisted with fieldwork. Collections in Vale Natural Reserve, Córrego do Veado Biological Reserve, Descobrimento National Park and Sooretama Biological Reserve were made with assistance from park authorities and permit from ICMBIO \#39704-7. Work in the MCZ was assisted by Adrian Magaña, Scout Leonard, Dylan Ryals, Whit Farnum and Amie Jones. Alyssa Hernandez produced SEMs. Harvard Bauer core staff helped with steps of library preparation and sequencing. Gideon Bradburd provided access to the development version of BEDASSLE2. The computations in this paper were run on the FASRC Cannon cluster supported by the FAS Division of Science Research Computing Group at Harvard University. The first author received a Jorge Paulo Lemann Fellowship for Research in Brazil. This work was funded by NSF DEB \#1355169 and \#1601356 and the William F. Milton Fund.

\section{DATA AVAILABILITY}

Demultiplexed Illumina raw reads are deposited in the NCBI SRA, BioProject PRJNA397912, accessions SRR12602029-SRR12602364 for insect samples and SRR12603820-SRR12603892 for plant samples. 
bioRxiv preprint doi: https://doi.org/101101/842153; this version posted October 9, 2020. The copyright holder for this preprint (which was not

\section{CODE AVAILABILITY}

All $R$, Python and bash scripts are available in the github repository

https://github.com/brunoasm/rad palm weevil, with most R code provided as

Rmarkdown notebooks including detailed annotation and visualization of intermediate steps. 


\section{REFERENCES}

1. Zhang Z-Q. 2011 Phylum Arthropoda von Siebold, 1848 In: Zhang, Z.-Q. (Ed.) Animal biodiversity: An outline of higher-level classification and survey of taxonomic richness. Zootaxa 3148, 99-103.

2. Stork NE, McBroom J, Gely C, Hamilton AJ. 2015 New approaches narrow global species estimates for beetles, insects, and terrestrial arthropods. Proc. Natl. Acad. Sci. 112, 7519-7523. (doi:10.1073/pnas.1502408112)

3. Futuyma DJ, Agrawal AA. 2009 Macroevolution and the biological diversity of plants and herbivores. Proc. Natl. Acad. Sci. 106, 18054-18061.

4. Farrell BD. 1998 "'Inordinate Fondness"' explained: Why are there so many beetles? Science. 281, 555-559. (doi:10.1126/science.281.5376.555)

5. Bascompte J. 2013 Adaptation, Fast and Endless. Science. 341, 130-131. (doi:10.1126/science.1239702)

6. Thompson JN, Segraves KA, Althoff DM. 2017 Coevolution and Macroevolution. In Evolutionary Developmental Biology (eds L Nuno de la Rosa, G Müller), pp. 113. Cham: Springer International Publishing. (doi:10.1007/978-3-319-330389_125-1)

7. Janz N. 2011 Ehrlich and Raven revisited: Mechanisms underlying codiversification of plants and enemies. Annu. Rev. Ecol. Evol. Syst. 42, 71-89. (doi:10.1146/annurev-ecolsys-102710-145024)

8. Maron JL, Agrawal AA, Schemske DW. 2019 Plant-herbivore coevolution and plant speciation. Ecology 100, 1-11. (doi:10.1002/ecy.2704)

9. Forister ML et al. 2015 The global distribution of diet breadth in insect herbivores. Proc. Natl. Acad. Sci. 112, 442-447. (doi:10.1073/pnas.1423042112)

10. Matsubayashi KW, Ohshima I, Nosil P. 2010 Ecological speciation in phytophagous insects. Entomol. Exp. Appl. 134, 1-27. (doi:10.1111/j.15707458.2009.00916.x)

11. Becerra JX. 2015 On the factors that promote the diversity of herbivorous insects and plants in tropical forests. Proc. Natl. Acad. Sci. U. S. A. 112, 6098-6103. (doi:10.1073/pnas.1418643112)

12. Schuman MC, Van Dam NM, Beran F, Harpole WS. 2016 How does plant chemical diversity contribute to biodiversity at higher trophic levels? Curr. Opin. Insect Sci. 14, 46-55. (doi:10.1016/j.cois.2016.01.003)

13. Ehrlich PR, Raven PH. 1964 Butterflies and plants: a study in coevolution. Evolution. 18, 586-608. 
14. McKenna DD et al. 2019 The evolution and genomic basis of beetle diversity. Proc. Natl. Acad. Sci. , 201909655. (doi:10.1073/pnas.1909655116)

15. Kopp M, Gavrilets S. 2006 Multilocus genetics and the coevolution of quantitative traits. Evolution. 60, 1321-1336.

16. Yoder JB, Nuismer SL. 2010 When does coevolution promote diversification? Am. Nat. 176, 802-17. (doi:10.1086/657048)

17. Wang IJ, Bradburd GS. 2014 Isolation by environment. Mol. Ecol. 23, 5649-5662. (doi:10.1111/mec.12938)

18. Hembry DH, Althoff DM. 2016 Diversification and coevolution in brood pollination mutualisms: windows into the role of biotic interactions in generating biological diversity. Am. J. Bot. 103, 1783-1792. (doi:10.3732/ajb.1600056)

19. Liu M, Zhang J, Chen Y, Compton SG, Chen XY. 2013 Contrasting genetic responses to population fragmentation in a coevolving fig and fig wasp across a mainland-island archipelago. Mol. Ecol. 22, 4384-4396. (doi:10.1111/mec.12406)

20. Tian E, Nason JD, MacHado CA, Zheng L, Yu H, Kjellberg F. 2015 Lack of genetic isolation by distance, similar genetic structuring but different demographic histories in a fig-pollinating wasp mutualism. Mol. Ecol. 24, 5976-5991. (doi:10.1111/mec.13438)

21. Bain A et al. 2016 Geographic structuring into vicariant species-pairs in a wideranging, high-dispersal plant-insect mutualism: the case of Ficus racemosa and its pollinating wasps. Evol. Ecol. 30, 663-684. (doi:10.1007/s10682-016-9836-5)

22. Smith CI, Godsoe WKW, Tank S, Yoder JB, Pellmyr O. 2008 Distinguishing coevolution from covicariance in an obligate pollination mutualism: Asynchronous divergence in Joshua tree and its pollinators. Evolution. 62, 2676-2687. (doi:10.1111/j.1558-5646.2008.00500.x)

23. Smith Cl, Tank S, Godsoe W, Levenick J, Strand E, Esque T, Pellmyr O. 2011 Comparative phylogeography of a coevolved community: Concerted population expansions in Joshua trees and four Yucca moths. PLoS One 6. (doi:10.1371/journal.pone.0025628)

24. Espíndola A, Carstens BC, Alvarez N. 2014 Comparative phylogeography of mutualists and the effect of the host on the genetic structure of its partners. Biol. J. Linn. Soc. 113, 1021-1035. (doi:10.1111/bij.12393)

25. Hembry DH, Kawakita A, Gurr NE, Schmaedick MA, Baldwin BG, Gillespie RG. 2013 Non-congruent colonizations and diversification in a coevolving pollination mutualism on oceanic islands. Proc. R. Soc. B Biol. Sci. 280, 20130361. (doi:10.1098/rspb.2013.0361) 
26. Thompson JN. 2005 The geographic mosaic of coevolution. Chicago: The University of Chicago Press.

27. Noblick LR. 2017 A revision of the genus Syagrus (Arecaceae). Phytotaxa 294, 001-262. (doi:10.11646/phytotaxa.294.1.1)

28. Meerow AW, Noblick LR, Salas-leiva DE, Sanchez V, Francisco-ortega J, Jestrow B, Nakamura K. 2015 Phylogeny and historical biogeography of the cocosoid palms (Areaceae, Arecoideae, Cocoseae) inferred from sequences of six WRKY gene family loci. Cladistics 31, 509-534. (doi:10.111/cla.12100)

29. Silberbauer-Gottsberger I, Vanin SA, Gottsberger G. 2013 Interactions of the Cerrado palms Butia paraguayensis and Syagrus petraea with parasitic and pollinating insects. Sociobiology 60, 306-316.

(doi:10.13102/sociobiology.v60i3.306-316)

30. Núñez-Avellaneda LA, Rojas-Robles R. 2008 Biología reproductiva y ecología de la polinización de la palma milpesos Oenocarpus bataua en los Andes colombianos. Caldasia 30, 101-125.

31. Núñez-Avellaneda LA, Isaza C, Galeano G. 2015 Ecología de la polinización de tres especies de Oenocarpus (Arecaceae) simpátricas en la Amazonia colombiana. Rev. Biol. Trop. 63, 35-55.

32. de Medeiros BAS, Núñez-Avellaneda LA, Hernandez AM, Farrell BD. 2019 Flower visitors of the licuri palm (Syagrus coronata): brood pollinators coexist with a diverse community of antagonists and mutualists. Biol. J. Linn. Soc. 126, 666687. (doi:10.1093/biolinnean/blz008)

33. Barbosa CM, Maia ACD, Martel C, Regueira JCS, do Amaral Ferraz Navarro DM, Raguso RA, Milet $\square$ Pinheiro P, Machado IC. 2020 Reproductive biology of Syagrus coronata (Arecaceae): sex $\square$ biased insect visitation and the unusual case of scent emission by peduncular bracts . Plant Biol. (doi:10.1111/plb.13162)

34. McKenna DD et al. 2018 Morphological and Molecular Perspectives on the Phylogeny, Evolution, and Classification of Weevils (Coleoptera: Curculionoidea): Proceedings from the 2016 International Weevil Meeting. Diversity 10, 64. (doi:10.3390/d10030064)

35. Souza MCP, Moura F, Silva J V., Almeida C. 2018 Phylogeography of the palm Syagrus coronata (Martius) Beccari (Arecaceae): distribution in the "Caatinga" and Atlantic forest domains. Rev. Bras. Bot. 41, 849-857. (doi:10.1007/s40415018-0498-0)

36. Peterson BK, Weber JN, Kay EH, Fisher HS, Hoekstra HE. 2012 Double Digest RADseq: An inexpensive method for de novo SNP discovery and genotyping in model and non-model species. PLoS One 7, e37135.

(doi:10.1371/journal.pone.0037135) 
37. de Medeiros BAS, Farrell BD. 2018 Whole-genome amplification in double-digest RADseq results in adequate libraries but fewer sequenced loci. PeerJ 6, e5089. (doi:10.7717/peerj.5089)

38. Bondar GG. 1940 Notas Entomológicas da Baía. VI. Rev. Entomol. 11, 842-861.

39. Bondar GG. 1941 Notas Entomológicas da Baía. VIII. Rev. Entomol. 12, 427-470.

40. Bondar GG. 1942 Notas Entomológicas da Baía. IX. Rev. Entomol. 13, 1-38.

41. Rubin BER, Ree RH, Moreau CS. 2012 Inferring phylogenies from RAD sequence data. PLoS One 7, e33394. (doi:10.1371/journal.pone.0033394)

42. Feder JL, Nosil P, Wacholder a. C, Egan SP, Berlocher SH, Flaxman SM. 2014 Genome-wide congealing and rapid transitions across the speciation continuum during speciation with gene flow. J. Hered. 105, 810-820.

(doi:10.1093/jhered/esu038)

43. Struck TH et al. 2017 Finding evolutionary processes hidden in cryptic species. Trends Ecol. Evol. 33, 153-163. (doi:10.1016/j.tree.2017.11.007)

44. GBIF.org. 2019 GBIF Ocurrence Download. (doi:10.15468/dl.Iprfwo)

45. Bradburd GS, Ralph PL, Coop GM. 2013 Disentangling the effects of geographic and ecological isolation on genetic differentiation. Evolution (N. Y). 67, 3258-73. (doi:10.1111/evo.12193)

46. Bradburd GS. 2020 BEDASSLE 2.0. Available at: https://github.com/gbradburd/bedassle

47. Weiblen GD, Treiber EL. 2015 Evolutionary origins and diversification of mutualism. In Mutualism (ed JL Bronstein), pp. 37-56. Oxford University Press. (doi:10.1093/acprof:oso/9780199675654.003.0003)

48. Kaur KM, Malé P-JG, Spence E, Gomez C, Frederickson ME. 2019 Using textmined trait data to test for cooperate-and-radiate co-evolution between ants and plants. PLOS Comput. Biol. 15, e1007323. (doi:10.1371/journal.pcbi.1007323)

49. Kawakita A, Okamoto T, Goto R, Kato M. 2010 Mutualism favours higher host specificity than does antagonism in plant-herbivore interaction. Proc. $R$. Soc. $B$ Biol. Sci. 277, 2765-2774. (doi:10.1098/rspb.2010.0355)

50. Guerrero-Olaya NY, Núñez-Avellaneda LA. 2017 Ecología de la polinización de Syagrus smithii (Arecaceae), una palma cantarofila de la Amazonia Colombiana. Rev. Peru. Biol. 24, 43-54. (doi:10.15381/rpb.v24i1.13102)

51. Thompson JN, Fernandez CC. 2006 Temporal dynamics of antagonism and mutualism in a geographically variable plant-insect interaction. Ecology 87, 103- 
12.

52. Uhl NW, Moore HE. 1973 The protection of pollen and ovules in palms. Principes 17, 111-150.

53. McKenna DD et al. 2019 The evolution and genomic basis of beetle diversity. Proc. Natl. Acad. Sci. U. S. A. 116, 24729-24737. (doi:10.1073/pnas.1909655116)

54. Hazzouri KM, Sudalaimuthuasari N, Kundu B, Nelson D, Al-Deeb MA, Le Mansour A, Spencer JJ, Desplan C, Amiri KMA. 2020 The genome of pest Rhynchophorus ferrugineus reveals gene families important at the plant-beetle interface. Commun. Biol. 3. (doi:10.1038/s42003-020-1060-8)

55. Vertacnik KL, Linnen CR. 2017 Evolutionary genetics of host shifts in herbivorous insects: insights from the age of genomics. Ann. N. Y. Acad. Sci. 1389, 186-212. (doi:10.1111/nyas.13311)

56. Forbes AA, Devine SN, Hippee AC, Tvedte ES, Ward AKG, Widmayer HA, Wilson CJ. 2017 Revisiting the particular role of host shifts in initiating insect speciation. Evolution (N. Y). 71, 1126-1137. (doi:10.1111/evo.13164)

57. Althoff DM, Segraves KA, Johnson MTJ. 2014 Testing for coevolutionary diversification: linking pattern with process. Trends Ecol. Evol. 29, 82-9. (doi:10.1016/j.tree.2013.11.003)

58. Hembry DH, Yoder JB, Goodman KR. 2014 Coevolution and the Diversification of Life. Am. Nat. 184, 425-438. (doi:10.1086/677928)

59. Jermy T. 1993 Evolution of insect $\square$ plant relationships $\square$ a devil's advocate approach. Entomol. Exp. Appl. 66, 3-12. (doi:10.1111/j.15707458.1993.tb00686.x)

60. Jermy T. 1984 Evolution of insect/host plant relationships. Am. Nat. 124, 609630.

61. Brand P, Hinojosa-Díaz IA, Ayala R, Daigle M, Yurrita Obiols CL, Eltz T, Ramírez SR. 2020 The evolution of sexual signaling is linked to odorant receptor tuning in perfume-collecting orchid bees. Nat. Commun. 11. (doi:10.1038/s41467-01914162-6)

62. Wardhaugh CW. 2015 How many species of arthropods visit flowers? Arthropod. Plant. Interact. 9, 547-565. (doi:10.1007/s11829-015-9398-4)

63. Eaton DAR. 2014 PyRAD: Assembly of de novo RADseq loci for phylogenetic analyses. Bioinformatics 30, 1844-1849. (doi:10.1093/bioinformatics/btu121)

64. Eaton DAR, Overcast I. 2019 ipyrad. Available at: https://ipyrad.readthedocs.io 
65. Anbutsu $\mathrm{H}$ et al. 2017 Small genome symbiont underlies cuticle hardness in beetles. Proc. Natl. Acad. Sci. , 201712857. (doi:10.1073/pnas.1712857114)

66. Xiao $\mathrm{Y}$ et al. 2017 The genome draft of coconut (Cocos nucifera). Gigascience 6, 1-11. (doi:10.1093/gigascience/gix095)

67. de Medeiros BAS. 2019 Matrix Condenser. Available at: https://github.com/brunoasm/matrix_condenser

68. Schliep KP. 2011 phangorn: phylogenetic analysis in R. Bioinformatics 27, 592-3. (doi:10.1093/bioinformatics/btq706)

69. Legendre P, Lapointe F-J, Casgrain P. 1994 Modeling brain evolution from behavior: A permutational regression approach. Evolution (N. Y). 48, 1487. (doi:10.2307/2410243)

70. Lichstein JW. 2007 Multiple regression on distance matrices: A multivariate spatial analysis tool. Plant Ecol. 188, 117-131. (doi:10.1007/s11258-006-9126-3)

71. Goslee SC, Urban DL. 2007 The ecodist package for dissimilarity-based analysis of ecological data. J. Stat. Softw. 22, 1-19.

72. Galili T. 2015 dendextend: An R package for visualizing, adjusting and comparing trees of hierarchical clustering. Bioinformatics 31, 3718-3720.

(doi:10.1093/bioinformatics/btv428)

73. Winter DJ. 2012 MMOD: An R library for the calculation of population differentiation statistics. Mol. Ecol. Resour. 12, 1158-1160. (doi:10.1111/j.17550998.2012.03174.x)

74. Jombart T. 2008 Adegenet: A R package for the multivariate analysis of genetic markers. Bioinformatics 24, 1403-1405. (doi:10.1093/bioinformatics/btn129)

75. Jombart T, Ahmed I. 2011 adegenet 1.3-1: New tools for the analysis of genomewide SNP data. Bioinformatics 27, 3070-3071.

(doi:10.1093/bioinformatics/btr521)

76. Hedrick PW. 2005 A standardized genetic differentiation measure. Evolution (N. Y). 59, 1633. (doi:10.1554/05-076.1)

77. Li H, Durbin R. 2009 Fast and accurate short read alignment with BurrowsWheeler transform. Bioinformatics 25, 1754-1760.

(doi:10.1093/bioinformatics/btp324)

78. Korneliussen TS, Albrechtsen A, Nielsen R. 2014 ANGSD: Analysis of Next Generation Sequencing Data. BMC Bioinformatics 15, 356. (doi:10.1186/s12859014-0356-4) 
79. Meisner J, Albrechtsen A. 2018 Inferring population structure and admixture proportions in low-depth NGS data. Genetics 210, 719-731.

(doi:10.1534/genetics.118.301336)

80. Meisner J, Albrechtsen A. 2019 Testing for Hardy-Weinberg equilibrium in structured populations using genotype or low-depth next generation sequencing data. Mol. Ecol. Resour. 19, 1144-1152. (doi:10.1111/1755-0998.13019)

81. Lee C, Abdool A, Huang CH. 2009 PCA-based population structure inference with generic clustering algorithms. BMC Bioinformatics 10, 1-13. (doi:10.1186/14712105-10-S1-S73)

82. Gutenkunst RN, Hernandez RD, Williamson SH, Bustamante CD. 2009 Inferring the joint demographic history of multiple populations from multidimensional SNP frequency data. PLoS Genet. 5. (doi:10.1371/journal.pgen.1000695)

83. Hey J, Nielsen R. 2004 Multilocus methods for estimating population sizes, migration rates and divergence time, with applications to the divergence of Drosophila pseudoobscura and D. persimilis. Genetics 167, 747-60. (doi:10.1534/genetics.103.024182)

84. Excoffier L, Foll M. 2011 fastsimcoal: A continuous-time coalescent simulator of genomic diversity under arbitrarily complex evolutionary scenarios. Bioinformatics 27, 1332-1334. (doi:10.1093/bioinformatics/btr124)

85. Excoffier L, Dupanloup I, Huerta-Sánchez E, Sousa VC, Foll M. 2013 Robust Demographic Inference from Genomic and SNP Data. PLoS Genet. 9. (doi:10.1371/journal.pgen.1003905)

86. Oppold A-M, Pfenninger M. 2017 Direct estimation of the spontaneous mutation rate by short-term mutation accumulation lines in Chironomus riparius . Evol. Lett. 1, 86-92. (doi:10.1002/evl3.8)

87. Guillot G, Schilling RL, Porcu E, Bevilacqua M. 2014 Validity of covariance models for the analysis of geographical variation. Methods Ecol. Evol. 5, 329335. (doi:10.1111/2041-210X.12167)

88. Pebesma E. 2018 Simple Features for R: Standardized Support for Spatial Vector Data. $R$ Journal. 10, 439-446. (doi:10.32614/RJ-2018-009)

89. Chamberlain S, Barve V, Mcglinn D, Oldoni D, Desmet P, Geffert L, Ram K. 2019 rgbif: Interface to the Global Biodiversity Information Facility API. Available at: https://cran.r-project.org/package=rgbif

90. Zizka A et al. 2019 CoordinateCleaner: Standardized cleaning of occurrence records from biological collection databases. Methods Ecol. Evol. 10, 744-751. (doi:10.1111/2041-210X.13152) 
91. Hijmans RJ. 2019 raster: Geographic Data Analysis and Modeling. Available at: https://cran.r-project.org/package=raster

92. Hijmans RJ, Cameron SE, Parra JL, Jones PG, Jarvis A. 2005 Very high resolution interpolated climate surfaces for global land areas. Int. J. Climatol. 25, 1965-1978. (doi:10.1002/joc.1276)

93. Vieira FG, Lassalle F, Korneliussen TS, Fumagalli M. 2016 Improving the estimation of genetic distances from Next-Generation Sequencing data. Biol. J. Linn. Soc. 117, 139-149. (doi:10.1111/bij.12511)

94. Dray S, Dufour AB. 2007 The ade4 package: Implementing the duality diagram for ecologists. J. Stat. Softw. 22, 1-20. (doi:10.18637/jss.v022.i04)

95. Gabry J. 2018 shinystan: Interactive Visual and Numerical Diagnostics and Posterior Analysis for Bayesian Models. Avialable at: https://mc-stan.org

96. Stan Development Team. $2019\{$ RStan\}: the $\{R\}$ interface to $\{$ Stan $\}$. Available at: https://mc-stan.org 